\title{
¿Por qué es importante aplicar Docencia Inversa? ¿cómo hacerlo?
}

\author{
N. Montanes ${ }^{\mathrm{a}}$, L. Sánchez-Nacher ${ }^{\mathrm{a}}$, O. Fenollar ${ }^{\mathrm{a}}$,
} D. Garcia-Sanoguera ${ }^{\mathrm{a}}$ y L. Quiles-Carrillo ${ }^{\mathrm{a}}$

${ }^{a}$ Grupo de Innovación de Prácticas Académicas (GIPA), Universitat Politècnica de València, Plaza Ferrándiz y Carbonell s/n, Alcoy, Alicante (ESPAÑA).

\begin{abstract}
The Flipped Learning methodology is so called because it is based on reversing the traditional scheme of the learning process. It offers a host of potential benefits, such as having more time to attend individually each student during their own class time, the opportunity to develop an active and collaborative learning, or the possibility that the student is who sets his own pace in the learning process, among many others. But it is also true that, like any other change, the application of this new methodology will imply the realization of an initial effort based on the replanning of the classes and the topics, as well as the preparation or reconversion of the teaching materials. The objective of this paper is to present methods, tools and technological resources used successfully in the application of the Flipped Learning methodology, in a subject of the Industrial Design Engineering and Product Development Degree, taught at the Universitat Politècnica de València (UPV). At the same time it also shows how it is possible to combine the application of the Flipped Learning methodology with the development and work of certain transversal competences of the UPV.
\end{abstract}

Keywords: Flipped Learning, methodology, active learning, collaborative learning, transversal competences.

\section{Resumen}

La aplicación de la metodología de Docencia Inversa, llamada así porque se basa en invertir el esquema tradicional del proceso de aprendizaje, ofrece un sinfín de beneficios potenciales, como por ejemplo disponer de más tiempo para atender individualmente a cada alumno durante el propio espacio de clase, la oportunidad de desarrollar un aprendizaje activo y colaborativo, o la posibilidad de que sea el alumno el que marque su propio ritmo en el proceso de aprendizaje, entre otros muchos. Pero también es cierto que, como cualquier cambio, la aplicación de esta nueva metodología va a llevar implícito la realización de un esfuerzo inicial en base a la replanificación de las clases y los temarios, así como en la preparación o reconversión de determinados materiales docentes. El objetivo del presente trabajo es presentar métodos, herramientas y recursos tecnológicos utilizados con éxito en la aplicación de la metodología de Docencia Inversa, en una asignatura 
del Grado en Ingeniería en Diseño Industrial y Desarrollo de Productos, impartida en la Universitat Politècnica de València (UPV). Al mismo tiempo también se expone cómo es posible combinar la aplicación de la metodología de Docencia Inversa con el desarrollo y trabajo de determinadas competencias transversales de la UPV.

Palabras clave: Docencia Inversa, metodología, aprendizaje activo, aprendizaje colaborativo, competencias transversales.

\section{Introducción}

En la actualidad una nueva forma metodológica de transmitir conocimientos, de desarrollar la tarea docente, de redirigir el aprendizaje, etc., está en pleno auge. Esta nueva metodología es la denominada Docencia Inversa, o en inglés Flipped Learning, y es llamada así porque se basa en invertir el esquema tradicional del proceso de aprendizaje (Roach, 2014).

Las largas clases magistrales con las que los profesores llenaban el tiempo del que disponían con sus alumnos en las aulas es liberado. Para ello, los profesores deben preparar materiales docentes adecuados para que los alumnos trabajen los contenidos de las antiguas clases magistrales en casa. Y una vez en el aula el tiempo es empleado para realizar toda una serie de actividades que refuercen los conocimientos trabajados en casa (Roach, 2014).

Según el equipo del Proyecto The Flipped Classroom, la metodología Flipped "trata de un enfoque integral que combina la instrucción directa con métodos constructivistas y el incremento de compromiso e implicación de los estudiantes con el contenido del curso y la mejora de su comprensión conceptual. Se trata de un enfoque integral que, cuando se aplica con éxito, apoya todas las fases de un ciclo de aprendizaje (Taxonomía de Bloom)” (Network, 2018).

Así pues, al quedar libre el tiempo de clase, los alumnos pueden participar en su propio aprendizaje de manera activa a través de actividades aplicadas, discusiones, debates, preguntas, etc. De esta manera se fomenta la exploración, la articulación y la aplicación de ideas en un ambiente de aprendizaje dinámico e interactivo (Network, 2018).

La metodología de Docencia Inversa ofrece muchos beneficios potenciales, como por ejemplo disponer de más tiempo para atender individualmente a cada alumno durante el propio espacio de clase, la oportunidad de desarrollar un aprendizaje activo y colaborativo, o la posibilidad de que sea el alumno el que marque su propio ritmo en el proceso de aprendizaje. Con esta metodología se puede conseguir un mejor uso del tiempo de clase para que los estudiantes participen en actividades que impliquen el desarrollo de estrategias de aprendizaje de nivel superior. Por otro lado, se promueve la participación de los estudiantes haciéndolos más visibles y se producen mejores interacciones entre los docentes y los alumnos, con un mayor feedback hacia los profesores (Bergmann and Sams, 2012; Bishop and Verleger, 2013; Roach, 2014; Network, 2018).

(cc) EY-NC-ND 2018, Universitat Politècnica de València

Congreso IN-RED (2018) 
Pero también es cierto que, como cualquier cambio, la metodología de Docencia Inversa va a llevar implícito la realización de un esfuerzo inicial en base a la replanificación de las clases y los temarios, así como en la preparación o reconversión de determinados materiales docentes, pero todos estos trabajos son asequibles y muchos los beneficios que se pueden lograr desde un punto de vista docente. Además en ningún momento se exige que una asignatura pase a ser trabajada mediante Docencia Inversa al $100 \%$ de un día para otro. Es más bien como un camino a recorrer, durante un determinado tiempo, pero con una meta clara.

Por último, cabe comentar que muchas de las herramientas y actividades empleadas en la metodología de Docencia Inversa permiten a su vez el desarrollo de las denominadas competencias transversales. En el marco del Espacio Europeo de Educación Superior (EEES) se ha producido un cambio de paradigma en el que la educación se está orientado a una formación basada en competencias. En el presente trabajo se expone también cómo es posible combinar la aplicación de la metodología de Docencia Inversa con el desarrollo y trabajo de determinadas competencias transversales de la Universitat Politècnica de València (UPV).

\section{Objetivos}

El objetivo del presente trabajo es presentar métodos, herramientas y recursos tecnológicos utilizados con éxito en la aplicación de la metodología de Docencia Inversa, en una asignatura del Grado en Ingeniería en Diseño Industrial y Desarrollo de Productos, impartida en la Universitat Politècnica de València (UPV). Se trata de la asignatura de Envase y Embalaje, del tercer curso, segundo cuatrimestre, que tiene una asignación de 4,5 créditos y 68 alumnos de media por curso.

La aplicación de dicha metodología va a requerir trabajar en dos frentes distintos: por un lado, se deberán establecer las actuaciones y materiales para el contexto casa y, por otro lado, se deberán plantear las actividades y materiales para el contexto aula.

En el contexto casa deben preparase una serie de materiales docentes que comprendan todos aquellos contenidos que en el método tradicional el profesor expone durante el tiempo de clase, mediante un discurso oral, apoyándose en presentaciones de transparencias, o en la pizarra. Para ello el docente puede elaborar videos explicativos de los contenidos, screencast, apuntes o recomendar lecturas interesantes, entre otras cosas.

Y de este modo, al quedar libre el tiempo de clase, en el contexto aula se pueden realizar por ejemplo actividades aplicadas, resolución de ejercicios, debates, exposiciones, juegos de preguntas y respuestas que refuercen los contenidos vistos en casa, etc. Lo importante es que los alumnos participen e interactúen y que el ambiente sea dinámico. En ese tiempo en el aula, el profesor puede atender de una manera más personal a cada uno de los alumnos y tener un feedback de cómo van avanzado en el aprendizaje de los contenidos de la asignatura, ya que no tiene que estar preocupándose de exponer contenidos teóricos. 
Además de todo esto, como se ha manifestado con anterioridad, en el presente trabajo también se va a exponer cómo es posible combinar la aplicación de la metodología de Docencia Inversa con el desarrollo y trabajo de determinadas competencias transversales de la UPV.

\section{Desarrollo de la innovación}

\subsection{Contexto casa}

El primer paso es analizar los contenidos que se desarrollan en la asignatura en cuestión, tras lo cual es posible decidir que partes, tanto de la teoría, como de la práctica, pueden trabajar los alumnos en casa con ayuda de determinados materiales docentes. Cabe comentar que la Universitat Politècnica de València cuenta con una serie de herramientas en línea para el aprendizaje. De todas esas herramientas se han escogido los objetos polimedia, los screencast y los artículos docentes como base para el desarrollo de los materiales a emplear en casa, para la aplicación de la Docencia Inversa, en la asignatura mencionada.

Los objetos polimedia son una grabación de un fragmento de 5 a 10 minutos de duración. En ellos se muestra, de forma sincronizada, la imagen y voz del profesor y una pantalla en la que se aparecen los contenidos de una presentación, una pizarra electrónica, un programa informático o una combinación de estos elementos. Este polimedia va dirigido a facilitar el aprendizaje del alumno de un concepto y/o procedimiento concreto, es decir, tiene una finalidad didáctica (Universitat Politècnica de València, 2018) (Fig. 1).
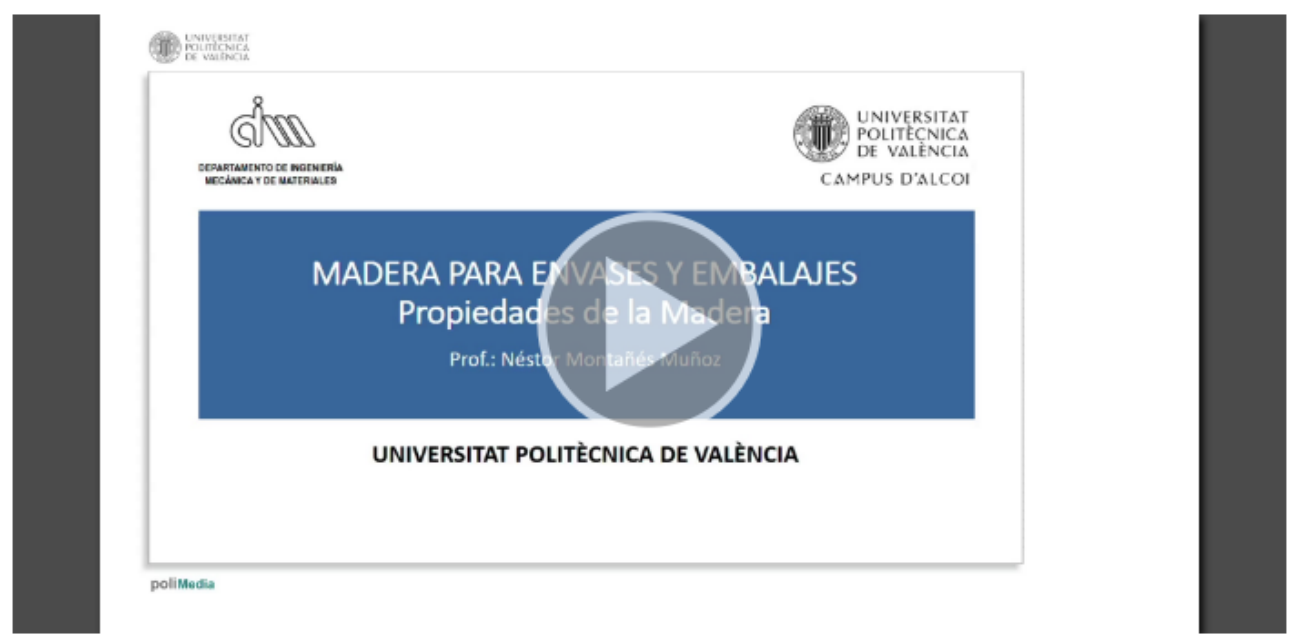

Fig. 1 Ejemplo de objeto polimedia

Por su parte los screencast son fragmentos de video educativo de una duración aproximada de entre 5 y 10 minutos. En ellos se muestran los contenidos generados mediante la captura de la pantalla del ordenador, acompañados de la voz en off del profesor o de un locutor. El screencast va dirigido a facilitar el aprendizaje del alumno de un concepto y/o procedimiento concreto, es decir, tiene una finalidad didáctica (Universitat Politècnica de 
València, 2018). Los screencast son ideales para enseñar el manejo de programas informáticos (Fig. 2).
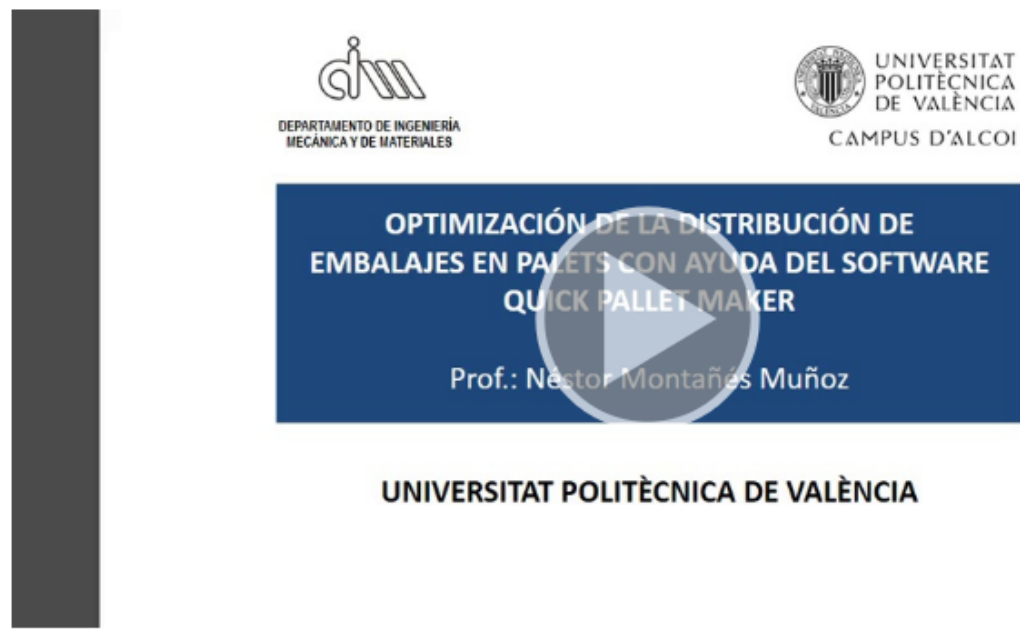

\section{UNIVERSITAT POLITÈCNICA DE VALÈNCIA}

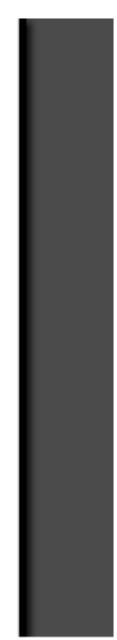

Fig. 2 Ejemplo de screencast

Por último, los artículos docentes son objetos de aprendizaje en formato texto digital, con una extensión entre 5 y 10 páginas, cuyo objetivo es facilitar el aprendizaje del alumno de un concepto y/o procedimiento concreto. Es decir, tiene una finalidad didáctica, por lo que debe ser motivador y enfocado al aprendizaje. No se tratan de artículos científicos, sino que su condición de objeto hace que sea algo totalmente aplicado y dirigido a guiar al alumno en su aprendizaje (Universitat Politècnica de València, 2018) (Fig. 3).

Así pues, se trata de ir preparando unidad a unidad, tema a tema, concepto a concepto, distintos materiales docentes con ayuda de estas herramientas en línea, siendo que en todo momento estos materiales docentes pueden ser complementados con, por ejemplo, apuntes elaborados por los profesores responsables de la asignatura, o con lecturas recomendadas de libros y artículos de revista. Pero tal y como se ha comentado anteriormente, este trabajo no tiene porque realizarse de golpe. El cambio de un método docente tradicional a la Docencia Inversa puede ser paulatino e ir realizándose a lo largo de los años. 


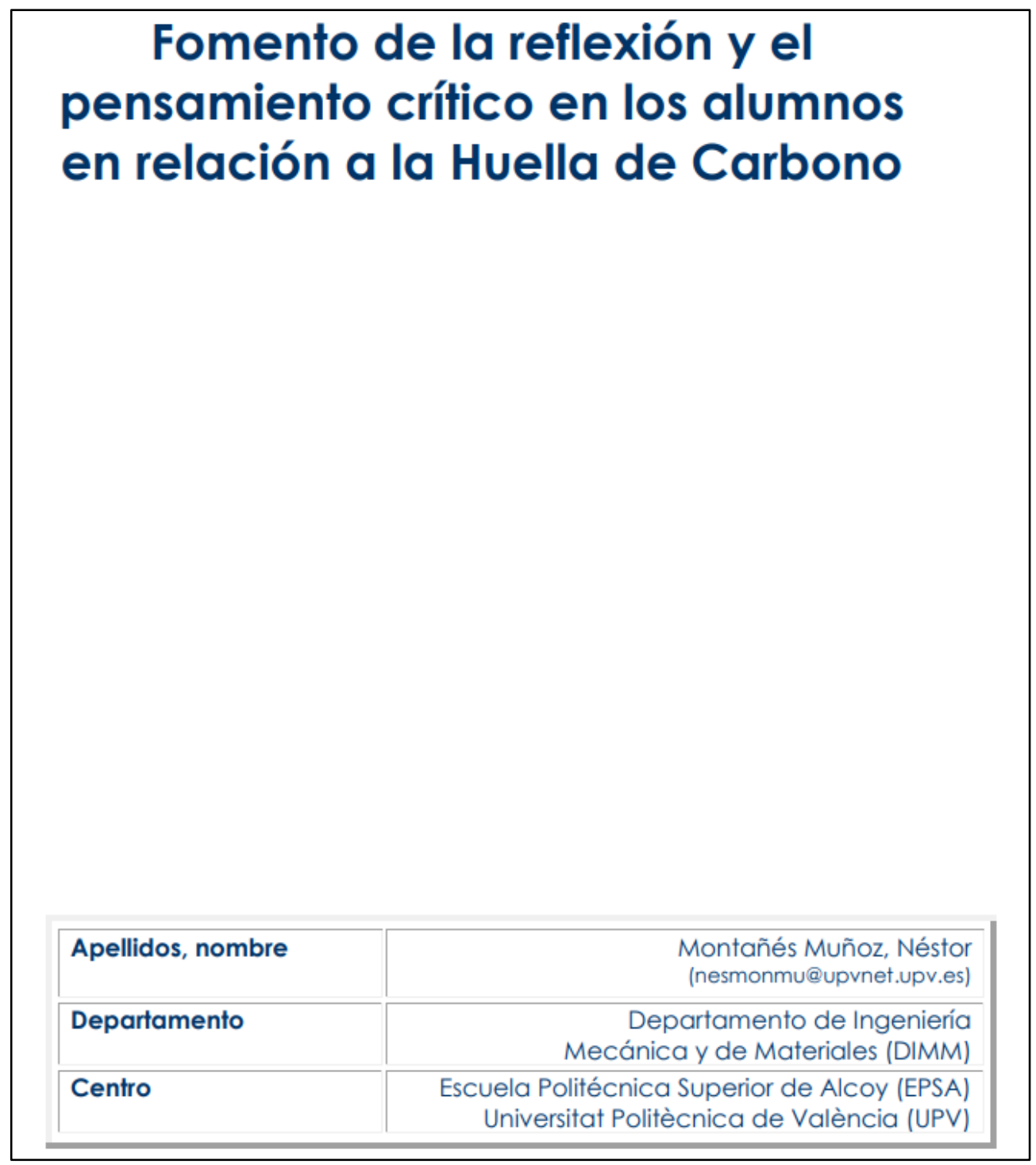

Fig. 3 Ejemplo de artículo docente

\subsection{Contexto clase.}

Una vez que los estudiantes han abordado los contenidos más teóricos de una sesión de clase, en casa, en el tiempo de clase es momento de realizar toda una serie de actividades que refuercen los conceptos adquiridos en casa. Por ejemplo, se pueden llevar a cabo actividades aplicadas, resolución de ejercicios, debates, exposiciones, juegos de preguntas/respuestas o desarrollo de pequeños proyectos, por citar algunas de las posibilidades.

A continuación se exponen ejemplos de algunas de estas actividades desarrolladas en el tiempo de clase de la asignatura Envase y Embalaje: 
- Actividades aplicadas: diseñar un envase de cartón o de hojalata para un frasco de perfume.

- Ejercicios / problemas: calcular cuantas unidades de un determinado envase caben en un palet normalizado y comparar los resultados obtenidos con los que muestra un programa informático que realiza este tipo de cálculos, cuyo manejo se ha aprendido en casa con ayuda de un screencast.

- Debates moderados: $\underline{\text { debatir }}$ de manera argumentada sobre los envases de plástico y el medio ambiente.

- Exposiciones: explicar ante el resto de los compañeros una parte de un tema. En el seno del Proyecto de competencias transversales de la UPV se encuentra la competencia “Comprensión e Integración”. Comprender quiere decir percibir y tener una idea clara de lo que se dice, se hace o sucede o descubrir el sentido profundo de algo. Para demostrar que algo se ha comprendido, la persona identifica y recupera la información y la explica con sus propias palabras, interpretando e integrando las ideas desde su propia perspectiva (Universitat Politècnica de València, 2018). Así pues, esta sería una actividad con la que además se desarrolla y trabaja una de las competencias transversales de la UPV.

- Proyectos: diseñar un envase de cartón para un frasco de perfume. Calcular el empaque óptimo en palets. Valorar el impacto medioambiental global del envase más el embalaje.

En los párrafos anteriores se han remarcado en negrita una serie de verbos, los cuales están en relación con la Taxonomía de Bloom. El objetivo es pues tratar de desarrollar actividades que promuevan el aprendizaje en los niveles superiores de dicha taxonomía. Lo importante es que los alumnos participen e interactúen, que el ambiente sea dinámico, que se refuercen los conocimientos trabajados en casa y que el docente se preocupe de tener un feedback continuo de la situación de cada alumno.

Por otro lado, también se pueden desarrollar actividades más lúdicas, como juegos grupales de preguntas y respuestas, en relación a un tema visto en casa. Así pues, son numerosos los métodos y herramientas con los que se puede desarrollar la Docencia Inversa en el aula.

\subsection{Ejemplo de aplicación en una unidad específica de la asignatura: Materiales de Madera para Envases y Embalajes.}

Como se ha comentado con anterioridad, el primer paso es analizar los contenidos que se desean desarrollar, así como los objetivos que se pretende que los alumnos alcancen. Tras ello se debe planificar y programar cada una de las sesiones, distribuir en el tiempo los materiales docentes y establecer cuáles serán las actividades a desarrollar en clase de manera que no sean repetitivas. Esta planificación inicial será necesario ir reprogramándola según avance el curso, con el feedback de se obtenga de los alumnos en el tiempo de clase. Un ejemplo de programación para una de estas sesiones puede ser el siguiente:

Polimedias: 
- Madera para Envases y Embalajes. Principios Generales. (Montañés, 2017) https://media.upv.es/player/?id=c8eb1be0-3595-11e7-8aa7-5b2740a8fd5f

- Madera para Envases y Embalajes. Propiedades de la Madera. (Montañés, 2017) https://media.upv.es/player/?id=dedb6950-3595-11e7-8aa7-5b2740a8fd5f

- Madera para Envases y Embalajes. Transformación de la Madera. (Montañés, 2018) https://media.upv.es/player/?id=6d1c4150-4a1f-11e8-af70-03116a2e3d4b

- Madera para Envases y Embalajes. Usos Específicos. (Montañés, 2018) https://media.upv.es/player/?id=76cbf600-4a1f-11e8-af70-03116a2e3d4b

- Madera para Envases y Embalajes. Palets. (Montañés, 2018) https://media.upv.es/player/?id=7f827da0-4a1f-11e8-af70-03116a2e3d4b

\section{Artículos:}

- ¿ ¿Cómo usando más madera se conservan los montes? (Plaza, 2016) http://www.maderea.es/usar-madera-y-conservar-los-montes/

- Toyota crea un coche de madera. (Maderea, 2016) http://www.maderea.es/toyota-crea-un-coche-de-madera/

- La madera transparente, ¿¿El fin del vidrio? (Maderea, 2016) http://www.maderea.es/madera-transparente/

- $\quad$ El fin del roble en España. (Parra, 2016) http://www.maderea.es/el-fin-del-roble-en-espana/

- Más madera contra el cambio climático. (Público, 2010) http://www.publico.es/ciencias/mas-madera-cambio-climatico.html

\section{Actividades en el aula:}

\section{Exposiciones:}

Se trata de que por grupos los alumnos expongan los contenidos de cada uno de los polimedias mencionados, asumiendo el rol de profesor. Se da un tiempo corto en clase para que preparen las exposiciones.

\section{Debates:}

La clase se divide en dos grandes grupos. Un grupo estará a favor de potenciar el uso de la madera en el sector del envase y el embalaje. El otro en contra. Apoyados en la información de los artículos entregados que previamente habrán leído en casa, así como en una pequeña búsqueda de información en clase, durante un tiempo cada grupo preparará sus argumentaciones. Tras ello cada grupo expondrá sus argumentos, tras lo cual comenzará un debate abierto moderado por el profesor. A los alumnos cuyas argumentaciones sean más sólidas y se apoyen en fuentes fiables, se les otorgará una puntuación extra a sumar en su nota final.

\subsection{Competencias transversales.}

Con las actividades comentadas en el apartado anterior del contexto clase se pueden trabajar de paso algunas de las 13 competencias transversales de la UPV. Por ejemplo, con

(c) ) EY-NC-ND 2018, Universitat Politècnica de València

Congreso IN-RED (2018) 
las exposiciones y los debates se puede desarrollar la competencia de "Comprensión e integración” como se ha visto. La resolución de actividades aplicadas y de ejercicios ayudarán a desarrollar la competencia "Análisis y resolución de problemas". El abordaje de pequeños proyectos colaborará en el desarrollo de la competencia "Diseño y proyecto”. Los juegos de preguntas y respuestas en grupos fomentarán la competencia de "Trabajo en equipo y liderazgo”. Los debates pueden ayudar a desarrollar un "Pensamiento crítico”.

De este modo, con una buena planificación de las actividades del tiempo de clase, sería posible trabajar prácticamente las 13 competencias transversales de la UPV.

\section{Resultados}

En el caso de la asignatura de Envase y Embalaje en la que se centra este artículo, la metodología de Docencia Inversa comenzó a aplicarse de manera paulatina a partir del curso 2015/2016. En el curso anterior, el 2014/2015, el método docente se basaba en las tradicionales clases magistrales.

Así pues, realmente solo se dispone de datos de dos cursos, ya que el curso actual, 2017/2018 todavía no ha acabado. Se ha analizado para estos dos cursos mencionados el rendimiento académico y la satisfacción de los alumnos registrada en las encuestas de evaluación de la docencia.

En los gráficos siguientes (Fig. 4 y Fig. 5), se puede observar como parece producirse un aumento en el rendimiento académico año tras año, reduciéndose el porcentaje de "No aptos" a apenas un $2 \%$ en el último curso, y como la satisfacción general de los alumnos, recogida en las encuestas de evaluación de la docencia, se ha incrementado en más de 1 punto respecto a cuándo el método docente que se empleaba eran las clases magistrales.

En cualquier caso, como se ha comentado, es pronto como para establecer conclusiones sólidas, ya que por ejemplo en relación a la mejora en el rendimiento académico, siendo tan grande el número de alumnos matriculados en la asignatura, las diferencias entre cursos pueden estar originadas por tan sólo uno o dos alumnos. 


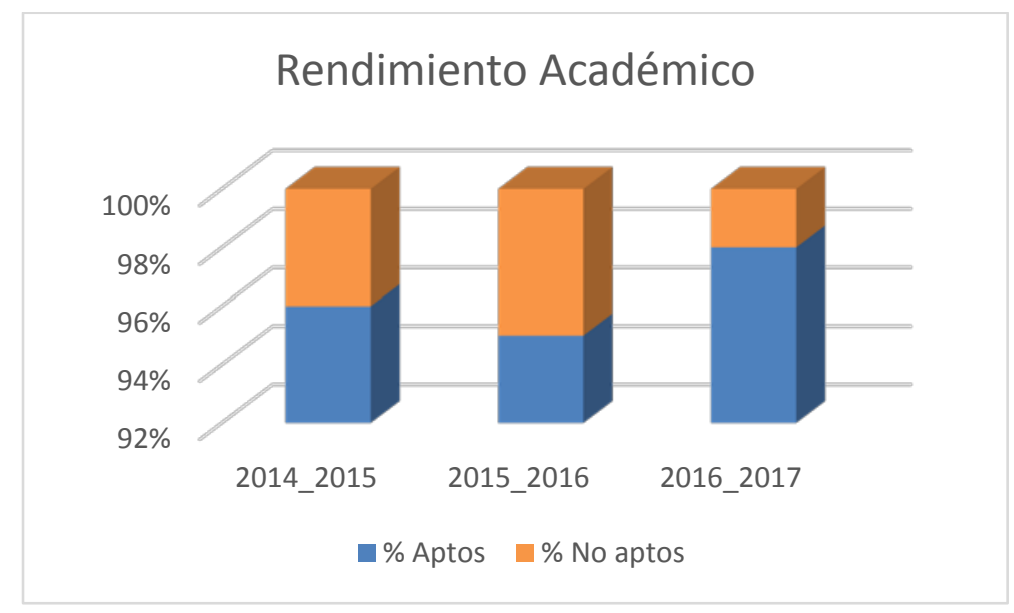

Fig. 4 Rendimiento académico de los alumnos de la asignatura de Envase y Embalaje de los últimos cursos

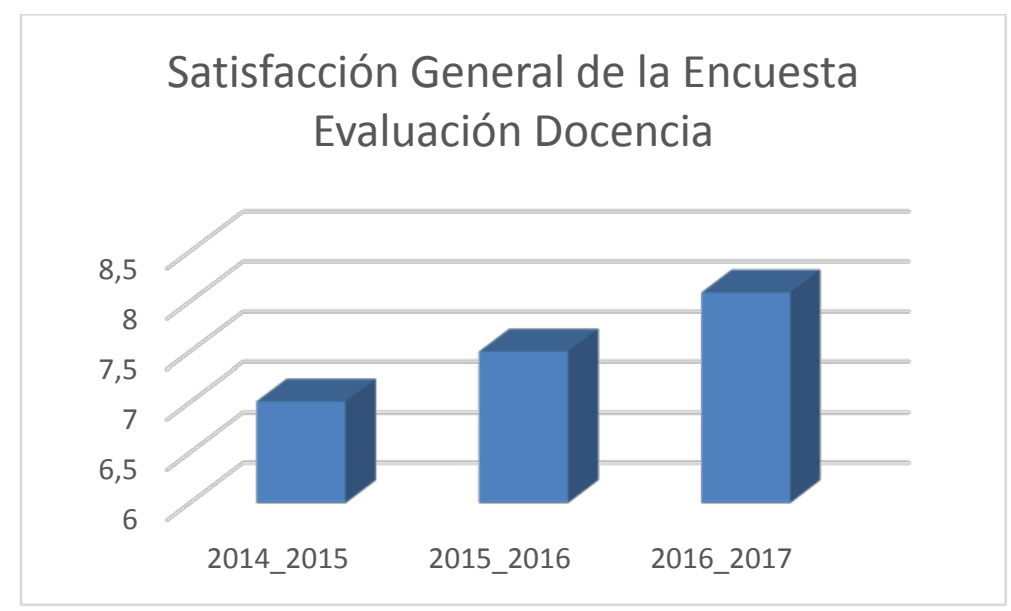

Fig. 5 Resultados de las encuestas de evaluación de la docencia para la asignatura de Envase y Embalaje de los últimos cursos

\section{Conclusiones}

Como se ha visto, la metodología de Docencia Inversa ofrece muchos beneficios potenciales en el proceso de aprendizaje de los alumnos. Si bien su aplicación requiere de un pequeño esfuerzo inicial por parte de los profesores, un replanteamiento de la asignatura y la elaboración de determinados materiales docentes, dichos beneficios mencionados a nivel pedagógico hacen que compense el esfuerzo a realizar por parte del profesorado.

En el presente trabajo se han expuesto a modo de ejemplo muchas herramientas y actividades con las que se puede realizar este cambio metodológico, tanto para el contexto casa (o fuera del aula), como para el contexto clase.

Por último, en relación a los resultados obtenidos de la aplicación del método de Docencia Inversa en la asignatura de "Envase y Embalaje", parece que se ha producido un aumento

(cc) BY-NC-ND 2018, Universitat Politècnica de València 
del rendimiento académico y se ha mejorado la satisfacción tanto de los alumnos, como de los profesores implicados, lo cual es muy positivo, aunque al disponer aún de pocos datos, no es posible establecer conclusiones muy firmes.

\section{Agradecimientos}

Este trabajo es fruto del esfuerzo que está realizando el Grupo de Innovación de Prácticas Académicas (GIPA) de la UPV en pro de la mejora docente. Los autores quieren mostrar su agradecimiento a la UPV y al Instituto de Ciencias de la Educación (ICE) de la UPV por su ayuda y colaboración en la formación del Equipo de Innovación y Calidad Educativa (EICE) denominado GIPA.

\section{Referencias}

Bergmann, J. and A. Sams (2012). Flip your classroom: Reach every student in every class every day, International Society for Technology in Education.

Bishop, J. L. and M. A. Verleger (2013). The flipped classroom: A survey of the research. ASEE National Conference Proceedings, Atlanta, GA.

Maderea, B. (2016). "La madera transparente, ¿El fin del vidrio?" Retrieved Junio 2018, from http://www.maderea.es/madera-transparente/.

Maderea, B. (2016). "Toyota crea un coche de madera." Retrieved Junio 2018, from http://www.maderea.es/toyota-crea-un-coche-de-madera/.

Montañés, N. (2017). "Madera para Envases y Embalajes. Principios Generales." Retrieved Junio 2018, from https://media.upv.es/player/? id=c8eb1be0-3595-11e7- 8aa7-5b2740a8fd5f.

Montañés, N. (2017). "Madera para Envases y Embalajes. Propiedades de la Madera." Retrieved Junio 2018, from https://media.upv.es/player/?id=dedb6950-3595-11e78aa7-5b2740a8fd5f.

Montañés, N. (2018). "Madera para Envases y Embalajes. Palets." Retrieved Junio 2018, from https://media.upv.es/player/?id=7f827da0-4a1f-11e8-af70-03116a2e3d4b.

Montañés, N. (2018). "Madera para Envases y Embalajes. Transformación de la Madera." Retrieved Junio 2018, from https://media.upv.es/player/? id=6d1c4150-4a1f-11e8-af70-03116a2e3d4b.

Montañés, N. (2018). "Madera para Envases y Embalajes. Usos Específicos." Retrieved Junio 2018, from https://media.upv.es/player/?id=76cbf600-4a1f-11e8-af7003116a2e3d4b.

Network, F. L. (2018). "What is the Flipped Classroom." Retrieved Marzo 2018, from https:/www.theflippedclassroom.es/what-is-innovacion-educativa/.

Parra, I. T. (2016). "El fin del roble en España." Retrieved Junio 2018, from http://www.maderea.es/el-fin-del-roble-en-espana/.

Plaza, B. S. (2016). "¿Cómo usando más madera se conservan los montes?" Retrieved Junio 2018, from http://www.maderea.es/usar-madera-y-conservar-los-montes/.

Público. (2010). "Más madera contra el cambio climático." Retrieved Junio 2018, from http://www.publico.es/ciencias/mas-madera-cambio-climatico.html. 
Roach, T. (2014). "Student perceptions toward flipped learning: New methods to increase interaction and active learning in economics." International Review of Economics Education 17: 74-84.

Universitat Politècnica de València, U. (2018). "Competencias Transversales UPV." Retrieved Marzo 2018, from http://www.upv.es/contenidos/COMPTRAN/.

Universitat Politècnica de València, U. (2018). "Docencia en Red: GUÍA PARA LA CREACIÓN DE ARTÍCULOS DOCENTES." Retrieved Marzo 2018, from http://www.upv.es/contenidos/DOCENRED/infoweb/docenred/info/PlantillaArticu losDocentes.dot.

Universitat Politècnica de València, U. (2018). "Docencia en Red: GUÍA PARA LA CREACIÓN DE GRABACIONES POLIMEDIA." Retrieved Marzo 2018, from http://www.upv.es/contenidos/DOCENRED/infoweb/docenred/info/guiaPolimedia scast.pdf.

Universitat Politècnica de València, U. (2018). "Docencia en Red: GUÍA PARA LA CREACIÓN DE GRABACIONES SCREENCAST." Retrieved Marzo 2018, from

http://www.upv.es/contenidos/DOCENRED/infoweb/docenred/info/U0759502.pdf 